BUHEP-99-2

YCTP-P2-99

SLAC-PUB-8045

\title{
A New Constraint on Strongly Coupled Field Theories
}

\author{
Thomas Appelquist ${ }^{a}$, Andrew G. Cohen ${ }^{b}$, and Martin Schmaltz ${ }^{c}$ \\ ${ }^{a}$ Department of Physics, Yale University, New Haven, CT 06511 \\ ${ }^{b}$ Department of Physics, Boston University, Boston, MA 02215
}

${ }^{c}$ SLAC, Stanford University, Stanford, CA 94309

January 24, 1999

\begin{abstract}
We propose a new constraint on the structure of strongly coupled field theories. The constraint takes the form of an inequality limiting the number of degrees of freedom in the infrared description of a theory relative to the number of underlying, ultraviolet degrees of freedom. We apply the inequality to a variety of theories (both supersymmetric and nonsupersymmetric), where it agrees with all known results and leads to interesting new constraints on low energy spectra. We discuss the relation of this constraint to Renormalization Group $c$-theorems.
\end{abstract}

*thomas.appelquist@yale.edu, cohen@bu.edu, schmaltz@slac.stanford.edu 


\section{Introduction}

Four dimensional field theories have been remarkably successful at describing nature at energies less than several hundred $\mathrm{GeV}$. Unfortunately progress at higher energies has been frustrated by a dearth of general theoretical tools that apply to strongly coupled models. Our understanding of field theory comes largely from perturbation theory (which applies to weakly coupled systems) and from QCD (where specific strong dynamics may be compared to experiment). There are many examples where this understanding is inadequate - for example, even the question of chiral symmetry breaking in a QCD-like gauge theory with a large number of flavors is unsettled $[1,2,3,4,5]$.

Recently new tools have appeared in the context of supersymmetric gauge theories. Known collectively as "duality", these ideas have produced convincing pictures of the pattern of symmetry breaking in many strongly coupled supersymmetric theories. The wide variety of low-energy phenomena that appear is remarkable, including dual gauge groups, conformal fixed points, chiral symmetry breaking, etc. These results are obtained without a detailed solution for the dynamics at strong coupling, but rely on symmetries, inspired guesswork, and general properties of supersymmetry. This shows that general constraints on the low energy properties of strongly coupled field theories are enormously useful, especially when a complete solution is unavailable.

The most powerful general constraint known is the anomaly matching 
condition introduced by 't Hooft [6]. Generally, we may define an anomaly as a residue of the pole in a particular multi-current correlation function. As discussed by 't Hooft, this number is independent of renormalization scale, and may therefore be computed at short distances, or equally well at long distances:

$$
\mathcal{A}_{I R}=\mathcal{A}_{U V}
$$

As the residue of a pole, the anomaly only receives contributions from physical massless degrees of freedom. If the short distance theory is weakly coupled (like an asymptotically free gauge theory) or calculable by other means, the anomaly condition provides an immediate relation of the massless spectrum to the short distance physics, constraining the appearance of massless fermions and Nambu-Goldstone bosons. Anomaly matching, as implementation of this condition is often called, has led to useful constraints on the possible low energy realizations of chiral gauge theories $[7,8]$, as well as QCD-like (vector-like) gauge theories [9, 10]. Anomalies have also played a fundamental role in discovering and checking the dualities of supersymmetric gauge theories [11, and references therein].

In this paper we propose a new constraint on the structure of strongly coupled field theories. Before stating and discussing this constraint, we note that, although the anomaly condition does not forbid the appearance of additional (vector-like) massless particles, it is usually assumed that the spectrum contains no massless particles that this condition does not require. That is, if there are relevant operators not forbidden by symmetries that would pro- 
duce masses, it is (technically) unnatural to assume that these operators are absent. Consequently we might say that nature generally abhors massless particles.

In fact when faced with the task of guessing the massless spectrum of a strongly coupled field theory, we are often guided by the idea that the number of massless particles is as small as possible. Since the anomaly condition can always be satisfied by a massless spectrum identical to the ultraviolet degrees of freedom, this would disfavor massless composites if the number of such composites is too large.

In elevating this casual notion to a formal principle, we need precise definitions of the number of degrees of freedom in both the infrared and ultraviolet. Although there are no unique such objects, we will choose to define quantities related to the free energy of the field theory. We will consider only renormalizable theories, for which the free energy may be rendered finite and cutoff independent by adjusting the vacuum energy to zero, renormalizing a finite set of parameters, and then removing the cutoff (holding physical quantities and the temperature fixed).

In terms of this properly renormalized free energy per unit volume, $\mathcal{F}$, (which is also equal to minus the pressure), the quantity that we will use to characterize the number of infrared degrees of freedom is

$$
f_{I R} \equiv-\lim _{T \rightarrow 0} \frac{\mathcal{F}}{T^{4}} \frac{90}{\pi^{2}}
$$

where $T$ is the temperature. For a free field theory, $f_{I R}$ is simply the number 
of massless bosons plus $7 / 8$ times the number of massless fermions. For an asymptotically free theory, the corresponding expression in the large $T$ limit mesaures the ultraviolet degrees of freedom in a similar way:

$$
f_{U V} \equiv-\lim _{T \rightarrow \infty} \frac{\mathcal{F}}{T^{4}} \frac{90}{\pi^{2}}
$$

Our qualitative discussion above suggests the new constraint $f_{I R} \leq f_{U V}$. In Section 2 we formulate this idea precisely and describe how this inequality (assuming that it is correct) leads to restrictions on the physical properties of strongly coupled field theories. Two examples are considered: a supersymmetric $S U(N)$ gauge theory with $F$ flavors, and a non-supersymmetric version of the same theory. In both cases the inequality will constrain the low energy structure. In Section 3 we describe a (failed) route to a proof of the inequality. The line of argument is nevertheless interesting, and leads to a deeper understanding of the inequality and its relation to so-called " $c$ theorems" [12]. In Section 4, we discuss the $T$ dependence for the two examples mentioned above. In Section 5 we apply the inequality to a variety of strongly coupled field theories. Finally, in Section 6 we summarize and conclude.

\section{The Inequality}

Our conjectured inequality is

$$
f_{I R} \leq f_{U V}
$$


subject to the condition that the limits in Eqs. $(2,3)$ exist. These limits are well defined when the theory has both a UV and an IR fixed point. Nothing in our discussion requires that either of these fixed points be free. However all our examples will involve asymptotically free gauge theories, and most will be infrared free as well.

In field theories with weakly coupled IR and UV fixed points the free energy, appearing in the definition of $f$, may be computed perturbatively. To zeroth order in couplings the low-temperature free energy density in three spatial dimensions is

$$
\mathcal{F}_{\text {free }}(T) \simeq-\frac{\pi^{2} T^{4}}{90}\left[N_{B}+\frac{7}{8} 2 N_{F}\right]
$$

where $N_{B}$ is the number of massless (real) bosonic fields, and $N_{F}$ is the number of massless (two-component) fermionic fields. We have neglected the contributions of any massive fields, which vanish exponentially as $T \rightarrow 0$. A similar expression applies in the infinite $T$ limit, with $N_{B}$ and $N_{F}$ including massive as well as massless fields. These expressions are exact in the case of free fixed points and approximately correct if the infrared or ultraviolet theories are governed by weak fixed points. For specific theories we may include perturbative corrections.

\subsection{SUSY Example}

For our first example we consider a SUSY $S U(N)$ gauge theory with $F$ flavors ("quarks" and "antiquarks") of massless fermions and associated superpartners. The theory has a free UV fixed point if the number of flavors is less 
than 3 times the number of colors, $F<3 N$. In this case the quantity $f_{U V}$ may be calculated using Eq. (5) to give

$$
f_{U V}=\left[2\left(N^{2}-1\right)+4 N F\right]\left(1+\frac{7}{8}\right) .
$$

The analysis of Seiberg [13] suggests that the infrared behavior of this theory is alternatively described through the use of $F$ flavors of massless magnetic quarks transforming according to the fundamental representation of a dual gauge group $S U(F-N)$, along with $F^{2}$ massless "meson" chiral superfields. This theory is infrared free provided $F \leq 3 N / 2$. Under these circumstances $f_{I R}$ is:

$$
f_{I R}=\left[2\left((F-N)^{2}-1\right)+4(F-N) F+2 F^{2}\right]\left(1+\frac{7}{8}\right) .
$$

Thus our fundamental inequality becomes

$$
2\left[(F-N)^{2}-1\right]+4(F-N) F+2 F^{2} \leq 2\left(N^{2}-1\right)+4 N F
$$

Because $f_{I R}$ grows quadratically with the number of flavors, this inequality limits the values of $F$ for which the low energy theory can consist of massless magnetic degrees of freedom with infrared free coupling. Remarkably, this inequality gives the bound $F \leq(3 / 2) N$, corresponding precisely to the boundary of the weak magnetic phase determined by the analysis of Seiberg [13]. At the boundary $F=(3 / 2) N$ the inequality is saturated ${ }^{1}$. We will show that the inequality continues to hold for $F>(3 / 2) N$ in Section 5.2 .

\footnotetext{
${ }^{1}$ The simplest example is the case $N=2: S U(2)$ gauge theory with 3 flavors. The theory confines and has an infrared-free dual description containing only a "meson" superfield, and $f_{U V}=f_{I R}=30(1+7 / 8)$.
} 


\section{$2.2 \quad$ Non-SUSY Example}

For our second example we consider the non-supersymmetric version of the same $S U(N)$ gauge theory, with $F$ massless quarks (and antiquarks). The theory has a free UV fixed point for $F<11 N / 2$. Based on real QCD we expect the $S U(F) \times S U(F)$ chiral symmetries of this theory to be realized in the Nambu-Goldstone mode - at least for small enough $F / N$. If we assume that this is the case, the IR theory consists of $F^{2}-1$ Nambu-Goldstone bosons. The derivative interactions of these particles are irrelevant in the infrared, and consequently this theory is described by a free IR fixed point.

At these free UV and IR fixed points we may use Eq. (5) to compute $f_{I R}$ and $f_{U V}$ :

$$
\begin{array}{r}
f_{I R}=F^{2}-1 \\
f_{U V}=2\left(N^{2}-1\right)+\frac{7}{8} 4 N F,
\end{array}
$$

and our inequality becomes

$$
F^{2}-1 \leq 2\left(N^{2}-1\right)+\frac{7}{8} 4 N F,
$$

or, since $F$ must be positive

$$
F \leq 4 \sqrt{N^{2}-\frac{16}{81}} .
$$

Since $N$ must be 2 or larger, and $F$ and $N$ must both be integral, this is equivalent to $F<4 N$. Remarkably, our inequality says that for the number of flavors larger than or equal to four times the number of colors, this gauge theory cannot break the full set of chiral symmetries! 
This new bound on the onset of the chiral phase transition $(F \leq 12$ for $S U(3))$ is well above the transitional values suggested by preliminary lattice simulations $[3,4]$. It is very close, however, to the value that emerges from the use of a continuum gap equation together with the assumption that the coupling is governed by an infrared fixed point appearing in the perturbative $\beta$ function [2]. In fact, a combination of the ladder gap equation and the two-loop beta function give a critical value $F^{c r i t} / N=\left(100 N^{2}-66\right) /\left(25 N^{2}-\right.$ $15)(\rightarrow 4$ as $N \rightarrow \infty)$. The reliability of this result is far from clear, however, since higher order effects are not obviously small. So whether the chiral phase transition saturates the inequality in this way or takes place at a lower value of $F / N$ remains an open question.

\section{Relation to $c$}

Having shown that the inequality Eq. (4) is consistent with other analyses of the SUSY $S U(N)$ theory and that it leads to a new result for QCD-like theories, we next discuss why it might be true generally. As an attempt at proof we may define a function $f(T)$ at all scales in an obvious way, as minus the free energy density divided by $T$ raised to the number of spatial dimensions plus one (this extension away from 4 space-time dimensions will prove useful shortly):

$$
f(T) \equiv-\frac{\mathcal{F}}{T^{d+1}} \Omega_{d}
$$

where $\Omega_{d}$ is a constant chosen such that the contribution to $f(T)$ from a free bosonic degree of freedom is 1 . The quantities $f_{I R}$ and $f_{U V}$ are just the limits 
of this function as $T$ approaches zero and $\infty$ respectively.

As a first step we differentiate the function $f$ with respect to $T$. Using the standard relations $T \partial \mathcal{F} / \partial T=-u-p, \mathcal{F}=-p$ where $p$ is the pressure and $u$ is the internal energy density, we have

$$
T \frac{\partial f}{\partial T}=\Omega_{d} \frac{u-d p}{T^{d+1}} \equiv \Omega_{d} \frac{\theta}{T^{d+1}}
$$

where $\theta$ is the (thermal average of the) trace of the energy-momentum tensor. For a conformally invariant theory, the trace of the energy-momentum tensor is zero. Under these circumstances we see that $f$ is a constant, and $f_{I R}$ is equal to $f_{U V}$. Of course the theories that we are interested in are not conformally invariant - the lack of conformal invariance arises from a scale dependence of coupling constants through renormalization. Consequently we expect the difference between $f_{U V}$ and $f_{I R}$ to arise from the renormalization group flow from the ultraviolet to the infrared. If $\theta$ is positive along this trajectory, $f_{I R}$ will necessarily be smaller than $f_{U V}$, proving our inequality.

Thus our inequality would follow from a positivity condition on the thermal average of the trace of the energy-momentum tensor; that is, from positivity of $u-d p$. For non-interacting systems, massive modes always have $p<u / d$ whereas massless modes have $p=u / d$. Even for interacting classical systems we expect these conditions to remain valid. Unfortunately the situation in quantum theories is not so simple $[14,15]$.

Consider, for example, a classically scale-invariant gauge field theory. In 
this case the trace of the energy-momentum tensor is given by:

$$
\theta=2 \frac{\beta}{g} \operatorname{Tr} G^{2}
$$

where $\operatorname{Tr} G^{2}$ is the trace over gauge indices of the square of the gauge fieldstrength, as well as over the thermal density matrix, and $\beta$ is the RG beta function. Note that, at least in perturbation theory, the thermal average of the field-strength squared is negative (magnetic fluctuations are less-well screened than electric fluctuations). Therefore a negative beta function leads to a positive $\theta$, as we desire.

Unfortunately this observation immediately suggests examples of negative $\theta$. If the low energy theory is a gauge field theory governed by a free infrared fixed point, then the $\beta$ function will be positive at weak coupling where $\operatorname{Tr} G^{2}$ is known to be negative. This is realized if the low energy theory is either an abelian theory with massless fermions or a non-abelian theory with matter content sufficient to render it infrared free. The SUSY $S U(N)$ theory in the weak magnetic phase, discussed in the previous section, is precisely such an example.

Of course this is not a counter-example to our conjectured inequality: the fact that $f(T)$ is not monotonic does not contradict the inequality involving $f_{I R}$ and $f_{U V}$. (We have already noted that the SUSY SU $(N)$ theory in the weak magnetic phase does in fact satisfy the inequality.) It means, however, that a proof of this inequality will be more involved than the simple argument used here. 
In addition to constraining the infrared behavior of field theories, the inequality can also restrict the general form of renormalization group flows. If we could construct a theory for which $\theta$ is always negative, the inequality would be violated. An example of such a theory might be a gauge theory in which $\theta$ is given by Eq. (14), $\beta$ is always positive, and the coupling is weak so that $\operatorname{Tr} G^{2}$ is always negative. Of course we continue to require a UV and an IR fixed point. We know of no example of a four dimensional gauge theory of this kind.

Note that had the function $f(T)$ been monotonic, we would have proven a " $c$-theorem": the existence of a function that is monotonic along RG trajectories. For example, in one spatial dimension the function $f(T)$ is monotonic, since the energy density is always greater than or equal to the pressure in the thermal state. But the value of this function at any fixed point (where $\theta=0)$ is simply the conventionally defined central charge of the corresponding conformal field theory ${ }^{2}$. The decrease in central charge between fixed points along an RG trajectory is the $c$-theorem of Zamolodchikov [12].

Our analysis implies that the existence of a corresponding $c$-theorem (with the same $c$ charge) in four space-time dimensions would lead to the inequality Eq. (4). If such a theorem does exist, the $c$-function is clearly not the function $f(T)$. Any other monotonic function that is equal to $f(T)$ at conformal fixed points is equally suitable for proving our conjecture. We have been

\footnotetext{
${ }^{2}$ Although our argument involves a flow in temperature, the usual RG arguments and dimensional analysis may be used to rewrite everything in terms of a change in scale parameter, $\mu$.
} 
unable to find such a function. Neither have we been able to establish the impossibility of such a function. Note that the existence of such a $c$-theorem, while providing a proof of Eq. (4), is not a necessary condition for the correctness of our much milder inequality.

There have been several attempts to prove a $c$-theorem in 4 dimensions $[16,17,18,19,20]$. The values of these $c$-functions at fixed points are numerically quite different from $f_{U V}$ and $f_{I R}$. The inequalities similar to Eq. (4) that would arise as consequences of these $c$-theorems in general do not significantly constrain the spectrum of 4 dimensional gauge field theories. The examples of Section 2 have already shown that our inequality does place interesting constraints on the spectrum of 4 dimensional gauge theories. Other examples will be presented in Section 5 .

\section{T Dependence}

We have stressed that the inequality Eq.(4) does not require the monotonicity of $f(T)$, and we have noted that for one example in which the inequality is satisfied (the supersymmetric $S U(N)$ theory in the weak magnetic phase), monotonicity is violated. In this section, we examine in more detail the $T$ dependence of $f(T)$ for this example and for the other example of section two: the non-supersymmetric $S U(N)$ theory. In each case, we record the $T$ dependence for both the $T \rightarrow \infty$ and $T \rightarrow 0$ limits, where perturbation theory may be employed. 


\subsection{SUSY $S U(N)$ Theory}

For $T \rightarrow \infty$, perturbation theory in the underlying, asymptotically free "electric" theory may be used, giving [21]

$$
f(T)=f_{U V}-\left(N^{2}-1\right)(N+3 F) \frac{45 g_{e}^{2}(T)}{32 \pi^{2}}+\ldots,
$$

where $f_{U V}$ is given by Eq. (6) and where $T$ sets the scale for the electric

coupling $g_{e}$. Since the $\beta$ function is negative, $g_{e}^{2}(T)$ decreases as $T$ increases, leading to positive $\theta$ and $\partial f / \partial T$, as discussed in Section 3.

For $T \rightarrow 0$, with $F \leq 3 N / 2$, perturbation theory gives

$$
f(T)=f_{I R}-\left((F-N)^{2}-1\right)(4 F-N) \frac{45 g_{m}^{2}(T)}{32 \pi^{2}}-3 F^{2}(F-N) \frac{45 y^{2}(T)}{32 \pi^{2}}+\ldots,
$$

where $f_{I R}$ is given by Eq. (7), $g_{m}$ is the magnetic gauge coupling, and $y$ is the Yukawa coupling of the magnetic theory. The $g_{m}^{2}$ term is obtained from the $g_{e}^{2}$ term in Eq. (15) by the replacement $N \rightarrow F-N$. The $y^{2}$ term is obtained by evaluating the two-loop diagrams involving the Yukawa couplings and the four-scalar couplings that arise from the superpotential of the magnetic theory.

Since the theory is infrared free for $F \leq 3 N / 2$, both couplings increase with $T$, showing that $\theta$ and $T \partial f / \partial T$ are negative for small $T$. Still, the inequality is satisfied.

\section{2 $S U(N)$ Theory}

We next record the $T$ dependence for the non-supersymmetric $S U(N)$ theory $[22, e g]$. For $T \rightarrow \infty$, perturbation theory gives 


$$
f(T)=f_{U V}-10\left(N^{2}-1\right)(N+5 F / N) \frac{g^{2}(T)}{16 \pi^{2}}+\ldots
$$

where $f_{U V}$ is given by Eq. (9) and $g$ is the gauge coupling. Asymptotic freedom leads to positive $\theta$ and $\partial f / \partial T$.

For $T \rightarrow 0$, corrections to the free-field behavior of the Nambu-Goldstone bosons may be computed using chiral perturbation theory. The leading correction arises at second order in $1 / F_{\pi}^{2}$, where $F_{\pi}$ is the Nambu-Goldstone decay constant, and contains a chiral logarithm. The result, for $T<<F_{\pi}$, is $[23]$

$$
f(T)=f_{I R}+\frac{F^{2}\left(F^{2}-1\right)}{144} \frac{T^{4}}{F_{\pi}^{4}} \ln \left(\frac{F_{\pi}}{T}\right)+\ldots,
$$

where $f_{I R}$ is given by Eq. (9). Thus for small $T, f(T)$ increases with $T$.

Interestingly, for the non-supersymmetric theory in the Nambu-Goldstone phase, the function $f(T)$ is positive-monotonic for both large $T$ and small $T$, the limits in which it may be computed reliably, using perturbation theory.

\section{Other Examples}

In this section we apply our inequality to several example field theories for which weakly coupled UV and IR descriptions have been proposed. We first discuss a number of additional asymptotically-free supersymmetric theories with infrared-free dual descriptions. We find the inequality to be satisfied in all cases. We then discuss the supersymmetric $S U(N)$ theory in the regime 
$F>3 N / 2$, where the dual magnetic theory exhibits a non-trivial infrared fixed point. The inequality is again satisfied where it can be checked perturbatively. Finally, we go on to discuss QED in $2+1$ dimensions, where the inequality gives an interesting constraint on the infrared spectrum.

\subsection{Infrared-Free Supersymmetric Examples}

All examples in this section are supersymmetric theories for which infraredfree dual descriptions have been proposed. We present each example in a for-

mat where we first describe the "electric" theory by giving its gauge group, matter content, and superpotential. We then give the gauge group and matter content of the dual "magnetic" description (and a reference to where this dual was first described in the literature). We show the range of flavors for which the magnetic description exists and is infrared-free. In each case, this is well within the flavor range for which the electric theory is asymptotically free. This is therefore the regime for which free field theory calculations of $f_{U V}$ and $f_{I R}$ are exact. We then quote the answers for $f_{U V}$ and $f_{I R}$ which are obtained by simply counting the number of superfields and multiplying them by a factor of $2(1+7 / 8)=15 / 4$, the contribution to the free energy from a single free superfield. Finally we compute the constraint following from $f_{I R} \leq f_{U V}$ and check whether it is satisfied in the range of flavors for which the calculation is valid. We find this to be the case in every example. A. The electric theory has $S O(N)$ gauge group with $F$ vectors and no tree level superpotential. The magnetic dual has gauge group $S O(F-N+4)$ with 
$F$ vectors and $F(F+1) / 2$ meson superfields [24]. As one can see from the following table the inequality is satisfied in the entire range of flavors where our calculation of the $f$ 's is applicable. Interestingly, as in the case of SUSY QCD the inequality is saturated at the boundary between the conformal and free phases of the dual description, which lies at $F=(3 / 2)(N-2)$.

\begin{tabular}{|c|c|}
\hline range of validity & $N-2 \leq F \leq \frac{3}{2}(N-2)$ \\
\hline$f_{U V}$ & $\frac{15}{4}\left[\frac{N(N-1)}{2}+F N\right]$ \\
\hline$f_{I R}$ & $\frac{15}{4}\left[\frac{(2 F-N+4)^{2}}{2}+\frac{N-4}{2}\right]$ \\
\hline inequality & $F \leq \frac{3}{2}(N-2)$ \\
\hline
\end{tabular}

B. The electric theory has $S p(2 N)$ gauge group with $2 F$ fundamentals and no tree level superpotential. The magnetic dual has gauge group $S p(2 F-2 N-4)$ with $2 F$ fundamentals and $F(2 F-1)$ mesons [25]. As one can see from the table the inequality is satisfied in the entire range of flavors where our calculation of the $f$ 's is applicable. As in the cases of $S O$ and $S U$ SUSY QCD, the inequality is saturated at the boundary between the conformal and free phases of the dual description, $F=(3 / 2)(N+1)$.

\begin{tabular}{|c|c|}
\hline range of validity & $N+3 \leq F \leq \frac{3}{2}(N+1)$ \\
\hline$f_{U V}$ & $\frac{15}{4}[N(2 N+1)+4 F N]$ \\
\hline$f_{I R}$ & $\frac{15}{4}\left[2(2 F-N-2)^{2}-N-2\right]$ \\
\hline inequality & $F \leq \frac{3}{2}(N+1)$ \\
\hline
\end{tabular}


C. The electric theory has $S U(N)$ gauge group with $F$ flavors and an adjoint chiral superfield $A$. Without a tree level superpotential no dual is known. With the superpotential $W=\operatorname{tr} A^{3}$ a magnetic dual has been found [26] with gauge group $S U(2 F-N)$. The matter content of this dual is: $F$ flavors of dual quarks, a chiral superfield transforming in the adjoint of the dual gauge group, and $2 F^{2}$ mesons $^{3}$. As we see from the table the inequality is satisfied in the entire range of flavors where our calculation of the $f$ 's is applicable.

\begin{tabular}{|c|c|}
\hline range of validity & $\frac{N}{2}<F \leq \frac{2}{3} N$ \\
\hline$f_{U V}$ & $\frac{15}{4}\left[2\left(N^{2}-1\right)+2 F N\right]$ \\
\hline$f_{I R}$ & $\frac{15}{4}\left[2\left(7 F^{2}-5 F N+N^{2}-1\right)\right]$ \\
\hline inequality & $F \leq \frac{6}{7} N$ \\
\hline
\end{tabular}

D. The electric theory has $S O(N)$ (or $S p(N)$ ) gauge group with $F$ vectors (fundamentals) ${ }^{4}$ and a symmetric (anti-symmetric) tensor $T$ of the gauge group. The tree level superpotential is $W=\operatorname{tr} T^{3}$. The magnetic dual [28] has gauge group $S O(2 F+8-N)(S p(2 F-8-N))$ with $F$ vectors (fundamentals), a symmetric (anti-symmetric) tensor, and $F(F \pm 1)$ mesons. Here and in the following the upper (lower) sign corresponds to the SO (Sp) model. Again we find that the inequality is satisfied in the entire range of flavors where our calculation of the f's is applicable.

\footnotetext{
${ }^{3}$ Note that there are also known duals [27] for more general superpotential terms $W=$ $\operatorname{tr} A^{k}$, but these theories do not have a weak UV fixed point so that we cannot calculate $f_{U V}$.

${ }^{4}$ In the case of $S p$ both $N$ and $F$ are even.
} 


\begin{tabular}{|c|c|}
\hline range of validity & $\frac{1}{2}(N+2 \mp 8) \leq F \leq \frac{2}{3}(N \mp 4)$ \\
\hline$f_{U V}$ & $\frac{15}{4}\left[N^{2}-1+F N\right]$ \\
\hline$f_{I R}$ & $\frac{15}{4}\left[7 F^{2}-5 F N+N^{2} \pm 41 F \mp 16 N+63\right]$ \\
\hline inequality & $N \geq \frac{7 F^{2} \pm 41 F+64}{6 F \pm 16}$ \\
\hline
\end{tabular}

E. In addition to the examples above we have also applied the predictions of the inequality to s-confining theories. These are $N=1$ SUSY gauge theories with no tree level superpotential which confine without chiral symmetry breaking. All s-confining theories have been identified and their IR spectra are known [29]. We find that the confined spectra for all these theories satisfy the inequality. Saturation occurs only for the s-confining $S U(2)$ theory with 3 flavors which we already mentioned in the footnote of section 2.1 .

\subsection{Supersymmetric Example with an Interacting In- frared Fixed Point}

We consider SUSY QCD for $F>(3 / 2) N$. Recall from section 2 that in this regime $f_{U V}$ is smaller than $f_{I R}$ computed at zero (magnetic) coupling. Thus it seems that our inequality might be violated. However, precisely at $F=(3 / 2) N$ the magnetic theory ceases to be infrared free and instead flows to an interacting fixed point. At this fixed point $f_{I R}$ receives corrections from the relevant interactions. These corrections are calculable in perturbation 
theory if the fixed point is weakly coupled and - as we will show below- are of the correct sign and magnitude to ensure that the inequality holds. These results are summarized in Figure 1. which shows $f_{U V}$ and $f_{I R}$ as a function of $F / N$ in the neighborhood of $F=(3 / 2) N$.

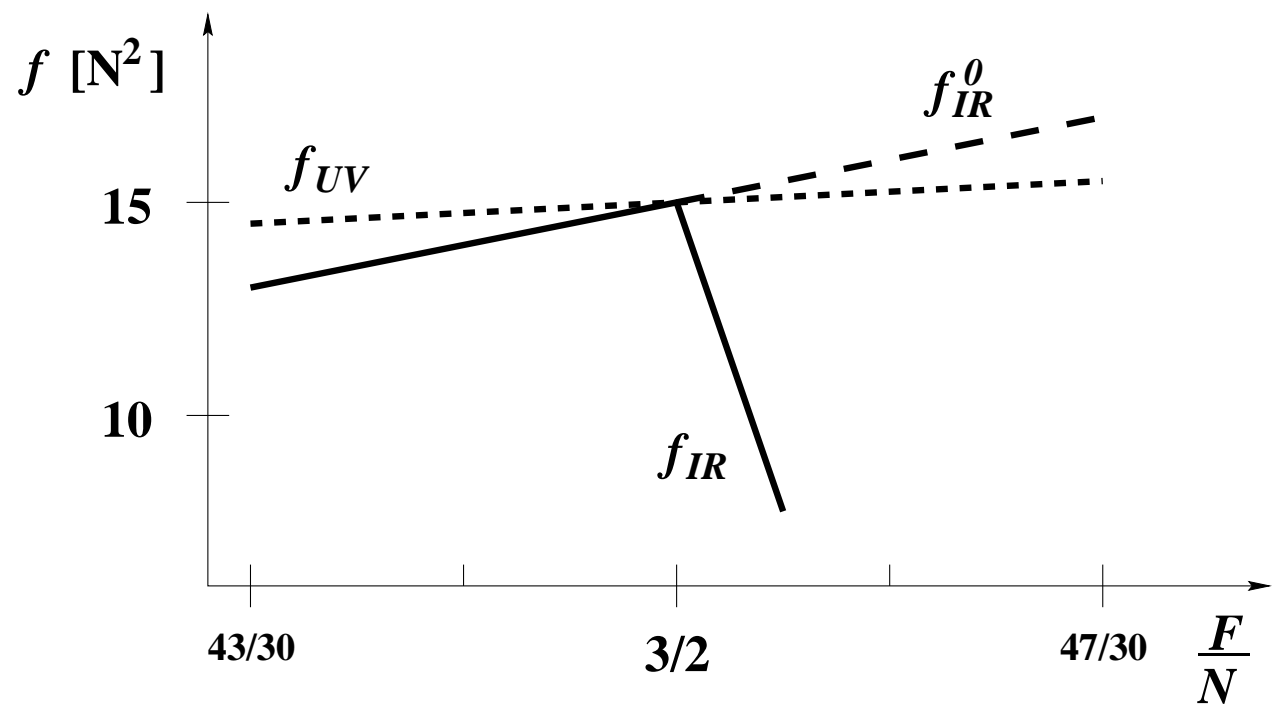

Figure 1: Plot of $f_{U V}$ and $f_{I R}$ in units of $N^{2}$ as functions of $F / N$. We have taken the large- $N$ and $F$ limit and show only the neighborhood of the interesting point $F / N=3 / 2$. For $F / N<3 / 2$ one sees that $f_{I R}<f_{U V}$, at $F / N=3 / 2$ the two $f$ 's touch, and for $F / N>3 / 2$ we again find $f_{I R}<f_{U V}$, but only after taking into account the interactions. For comparison we also show $f_{I R}^{0}$, the expression for $f_{I R}$ with no interactions included.

To calculate the corrections we choose large $N$ and $F$ with $F$ tuned slightly larger than $(3 / 2) N$. To see that for these values of $N$ and $F$ the fixed point of the magnetic theory is perturbative, define the small parameter $\epsilon \equiv(2 F-3 N) / N$ which measures the departure (in $F$ ) from the free magnetic phase. Then the fixed point values for the couplings of the magnetic theory 
may be computed in terms of $\epsilon$ by setting the two-loop $\beta$ functions for the gauge and Yukawa couplings to zero. We find

$$
\begin{aligned}
g_{m}^{2} & =16 \pi^{2} \frac{14}{3} \frac{\epsilon}{N} \\
y^{2} & =\frac{2}{7} g_{m}^{2}
\end{aligned}
$$

and one sees that perturbation theory in $g_{m}$ and $y$ holds as long as $\epsilon \ll 1$.

We now check that the inequality is also satisfied in this interacting theory by computing and comparing $f_{U V}$ and $f_{I R}$ at small $\epsilon$. Equation (6) expanded to first order in $\epsilon$ gives

$$
f_{U V}=15 N^{2}\left(1+\frac{1}{4} \epsilon\right)
$$

$f_{I R}$ receives contributions of order $\epsilon$ from expanding the free theory result Eq. (7) as well as from interactions. The interaction contribution is easily obtained from Eq. (16) by setting $g_{m}^{2}(T)$ and $y^{2}(T)$ equal to their fixed point values at $T=0$, Eq. (19). We obtain

$$
f_{I R}=15 N^{2}\left(1+\epsilon-\frac{31}{2} \epsilon\right)
$$

where the $+\epsilon$ comes from expanding the free expression whereas the $-\frac{31}{2} \epsilon$ comes from the interactions. Thus we see that our inequality $f_{U V} \geq f_{I R}$ is satisfied once the interactions are taken into account.

\subsection{QED in $\mathrm{d}=3$}

For 2+1 dimensional QED $\left(\mathrm{QED}_{3}\right)$ we will show that the inequality gives an interesting constraint on the allowed infrared phase structure. $\mathrm{QED}_{3}$ with 
$2 F$ charged Weyl fermions ( $F$ Dirac fermions) is believed to have a phase transition as the number of flavors is varied [30, 31]. The massless theory has a $U(2 F)$ global symmetry. For large $F$, the screening effect of the fermions prevents the formation of a condensate and the infrared theory is expected to be conformal. For small $F$, on the other hand, one expects global symmetry breaking and dynamical mass generation for the fermions. An analysis of the breaking using a gap equation indicates that a parity conserving mass term is formed, corresponding to the breaking of the global $U(2 F)$ symmetry to its $U(F) \times U(F)$ subgroup.

The inequality places a tight constraint on this pattern of breaking. $\mathrm{QED}_{3}$ is free in the ultraviolet and using Eq. (12) we have ${ }^{5}$

$$
f_{U V}=1+\frac{3}{4} 4 F
$$

where $4 F$ counts the fermionic degrees of freedom. The breaking of the $U(2 F)$ symmetry to $U(F) \times U(F)$ leads to $2 F^{2}$ Nambu-Goldstone bosons. Since the theory does not confine, the photon remains in the infrared spectrum so we have

$$
f_{I R}=1+2 F^{2}
$$

The inequality is satisfied only for $F \leq 3 / 2$ which implies that chiral symmetry breaking is excluded for all $F \geq 2$.

The critical number of flavors separating the two phases has been estimated, using the gap equation with a $1 / F$ expansion of the kernel, to be in

\footnotetext{
${ }^{5} \operatorname{In}(2+1)$ dimensions free bosons and fermions respectively contribute 1 and $3 / 4$ to $f$.
} 
the range $3<F_{\text {crit }}<4[30,31]$. The discrepancy between this result and our inequality suggests that the gap equation over-estimates $F_{\text {crit }}$.

\section{Conclusion}

We have considered a general constraint on the structure of strongly coupled field theories, the inequality Eq. (4). Although we have not proven this inequality, we have shown that it agrees with a large number of known results. In addition it places interesting restrictions on the pattern of symmetry breaking in many cases. The inequality (or one similar to it) would arise as a consequence of a $c$-theorem in four dimensions, but it is a weaker condition, and can be true even in circumstances where a $c$-theorem is not. It nevertheless provides a constraint on the general character of renormalization group flows for a wide variety of renormalizable field theories with IR and UV fixed points. It is also possible that the inequality applies not to all such field theories, but only a subset (e.g. asymptotically free theories). In specific cases it may be possible to prove the inequality via the route of section 3 .

Finally, it is interesting to apply the inequality to chiral gauge theories. In particular, in a model due to Bars and Yankielowicz [32] in which the anomaly matching conditions are consistent with the formation of massless composite fermions, the inequality leads to a nontrivial constraint on the infrared spectrum. In a future paper [33, in preparation], we will discuss the application of the inequality to this and several other chiral gauge models. 


\section{Acknowledgments}

We acknowledge the hospitality of the Aspen Center for Physics where this work was initiated. One of us (TA) also acknowledges the hospitality of Fermilab where he visited as a Frontier Fellow during October and November, 1998. We thank Nima Arkani-Hamed, Sidney Coleman, Erik D'Hoker, Dan Freedman, Subir Sachdev, Myckola Schwetz, Robert Shrock, R. Shankar and John Terning for helpful conversations. This work was supported in part by the Department of Energy under grant numbers DOE grant DE-FG0291ER40676, DE-FG02-92ER-40704 and DE-AC03-76SF00515.

\section{References}

[1] T. Appelquist, J. Terning, and L. C. R. Wijewardhana, "The Zero Temperature Chiral Phase Transition in SU(N) Gauge Theories," Phys. Rev. Lett. 77 (1996) 1214-1217, hep-ph/9602385.

[2] T. Appelquist, A. Ratnaweera, J. Terning, and L. C. R. Wijewardhana, "The Phase Structure of an SU(N) Gauge Theory with N(F) Flavors," Phys. Rev. D58 (1998) 105017, hep-ph/9806472.

[3] D. Chen and R. D. Mawhinney, "Dependence of QCD Hadron Masses on the Number of Dynamical Quarks," Nucl. Phys. Proc. Suppl. 53 (1997) 216, hep-lat/9705029.

[4] R. D. Mawhinney, "Evidence for Pronounced Quark Loop Effects in QCD," Nucl. Phys. Proc. Suppl. 60A (1998) 306, hep-lat/9705031. 
[5] E. Shuryak and T. Schafer, "Instantons and Chiral Symmetry Restoration in QCD-Like Theories," Nucl. Phys. Proc. Suppl. 53 (1997) 472.

[6] G. 't Hooft, "Naturalness, Chiral Symmetry, and Spontaneous Chiral Symmetry Breaking," in Recent Developments In Gauge Theories, G. 't Hooft et. al., eds., vol. 59 of NATO Advanced Study Institute, Series B: Physics. 1980.

[7] M. E. Peskin, "Chiral Symmetry and Chiral Symmetry Breaking," in Recent Advances in Field Theory and Statistical Mechanics, J. B. Zuber and R. Stora, eds., vol. 39 of Les Houches Summer School Proceedings. North-Holland, 1984.

[8] E. Eichten, R. D. Peccei, J. Preskill, and D. Zeppenfeld, "Chiral Gauge Theories in the 1/N Expansion," Nucl. Phys. B268 (1986) 161.

[9] S. Coleman and E. Witten, "Chiral Symmetry Breakdown in Large N Chromodynamics," Phys. Rev. Lett. 45 (1980) 100.

[10] C. Vafa and E. Witten, "Restrictions on Symmetry Breaking in Vector - Like Gauge Theories," Nucl. Phys. B234 (1984) 173.

[11] K. Intriligator and N. Seiberg, "Lectures on Supersymmetric Gauge Theories and Electric - Magnetic Duality," Nucl. Phys. Proc. Suppl. 45BC (1996) 1-28, hep-th/9509066. 
[12] A. B. Zamolodchikov, "Irreversibility' of the Flux of the Renormalization Group in a 2-D Field Theory," JETP Lett. 43 (1986) 730-732.

[13] N. Seiberg, "Electric - Magnetic Duality in Supersymmetric NonAbelian Gauge Theories," Nucl. Phys. B435 (1995) 129-146, hep-th/9411149.

[14] A. H. Castro Neto and E. Fradkin, "The Thermodynamics of Quantum Systems and Generalizations of Zamolodchikov's C Theorem," Nucl. Phys. B400 (1993) 525-546, cond-mat/9301009.

[15] S. Sachdev, "Polylogarithm Identities in a Conformal Field Theory in Three-Dimensions," Phys. Lett. B309 (1993) 285-288, hep-th/9305131.

[16] J. L. Cardy, "Is There a C Theorem in Four-Dimensions?," Phys. Lett. B215 (1988) 749 .

[17] I. Jack and H. Osborn, "Analogs for the C Theorem for Four-Dimensional Renormalizable Field Theories," Nucl. Phys. B343 (1990) 647.

[18] F. Bastianelli, "Tests for C Theorems in 4-D," Phys. Lett. B369 (1996) 249-254, hep-th/9511065.

[19] D. Z. Freedman and H. Osborn, "Constructing a C Function for SUSY Gauge Theories," Phys. Lett. B432 (1998) 353-360, hep-th/9804101. 
[20] S. Forte and J. I. Latorre, "A Proof of the Irreversibility Of Renormalization Group Flows in Four-Dimensions," Nucl. Phys. B535 (1998) 709, hep-th/9805015.

[21] J. Grundberg, T. H. Hansson, and U. Lindstrom, "Thermodynamics of N=1 Supersymmetric QCD," hep-th/9510045.

[22] A. V. Smilga, "Physics of Hot and Dense QCD," hep-ph/9901225.

[23] P. Gerber and H. Leutwyler, "Hadrons Below the Chiral Phase Transition," Nucl. Phys. B321 (1989) 387.

[24] K. Intriligator and N. Seiberg, "Duality, Monopoles, Dyons, Confinement and Oblique Confinement in Supersymmetric SO $(\mathrm{N}(\mathrm{C})$ ) Gauge Theories," Nucl. Phys. B444 (1995) 125-160, hep-th/9503179.

[25] K. Intriligator and P. Pouliot, "Exact Superpotentials, Quantum Vacua and Duality in Supersymmetric SP(N(C)) Gauge Theories," Phys. Lett. B353 (1995) 471-476, hep-th/9505006.

[26] D. Kutasov, "A Comment on Duality in N=1 Supersymmetric NonAbelian Gauge Theories," Phys. Lett. B351 (1995) 230-234, hep-th/9503086.

[27] D. Kutasov and A. Schwimmer, "On Duality in Supersymmetric Yang-Mills Theory," Phys. Lett. B354 (1995) 315-321, hep-th/9505004. 
[28] K. Intriligator, "New RG Fixed Points and Duality in Supersymmetric $\mathrm{SP}(\mathrm{N}(\mathrm{C}))$ and $\mathrm{SO}(\mathrm{N}(\mathrm{C}))$ Gauge Theories," Nucl. Phys. B448 (1995) 187-198, hep-th/9505051.

[29] C. Csaki, M. Schmaltz, and W. Skiba, "Confinement in N=1 SUSY Gauge Theories and Model Building Tools," Phys. Rev. D55 (1997) 7840-7858, hep-th/9612207.

[30] T. Appelquist, D. Nash, and L. C. R. Wijewardhana, "Critical Behavior in (2+1)-Dimensional QED," Phys. Rev. Lett. 60 (1988) 2575 .

[31] T. Appelquist, J. Terning, and L. C. R. Wijewardhana, "(2+1)-Dimensional QED and a Novel Phase Transition," Phys. Rev. Lett. 75 (1995) 2081-2084, hep-ph/9402320.

[32] I. Bars and S. Yankielowicz, "Composite Quarks and Leptons as Solutions of Anomaly Constraints," Phys. Lett. 101B (1981) 159.

[33] T. Appelquist, A. G. Cohen, M. Schmaltz, and R. Shrock, "New Constraints on Chiral Gauge Theories." in preparation. 